\title{
On Measures of Dependence Between Possibility Distributions
}

\author{
Robert Fullér ${ }^{1}$, István Á. Harmati ${ }^{2}$, Péter Várlaki ${ }^{3}$ \\ ${ }^{1}$ Department of Informatics \\ Széchenyi István University, Egyetem tér 1, H-9026, Győr, Hungary \\ e-mail: rfuller@sze.hu \\ ${ }^{2}$ Department of Mathematics and Computational Sciences \\ Széchenyi István University, Egyetem tér 1, H-9026, Győr, Hungary \\ e-mail: harmati@sze.hu \\ ${ }^{3}$ System Theory Lab \\ Széchenyi István University, Egyetem tér 1, H-9026, Győr, Hungary \\ e-mail:varlaki@sze.hu
}

\begin{abstract}
A measure of possibilistic correlation between marginal possibility distributions of a joint possibility distribution can be defined as (see Fullér, Mezei and Várlaki, An improved index of interactivity for fuzzy numbers, Fuzzy Sets and Systems, 165(2011), pp. 56-66) the weighted average of probabilistic correlations between marginal probability distributions whose joint probability distribution is defined to be uniform on the level sets of their joint possibility distribution. Using the averaging technique we shall discuss three quantities (correlation coefficient, correlation ratio and informational coefficient of correlation) which are used to measure the strength of dependence between two possibility distributions. We discuss the inverse problem, as we introduce a method to construct a joint possibility distribution for a given value of possibilistic correlation coefficient. We also discuss a special case when the joint possibility distribution is defined by the so-called weak t-norm and based on these results, we make a conjecture as an open problem for the range of the possibilistic correlation coefficient of any t-norm based joint distribution.
\end{abstract}

Keywords: possibility theory, fuzzy numbers, possibilistic correlation, possibilistic dependence.

\section{Introduction}

Random variables, probability distributions are widely used models of incomplete information [23], and measuring dependence between random variables and random sequences is one of the main tasks of applied probabilty and statistics. There are plenty of measures of dependence, for example correlation coefficients, correlation ration, distance correlation etc.

Possibility distributions are used to model human judgments and preferences and in this way they are models of non-statistical uncertainties. Measuring the strength of dependence between these non-statistical uncertain quantities is quite important, also 
from theoretical and practical point of view. In probability theory, measures of dependence are usually defined by using the expected value of an appropriate function of the random variables. In possibility theory a measure of possibilistic correlation between marginal possibility distributions of a joint possibility distribution can be defined as the weighted average of probabilistic measures of dependence between marginal probability distributions (fuzzy numbers) whose joint probability distribution is defined to be uniform on the $\gamma$-level sets (a.k.a $\alpha$-cuts) of their joint possibility distribution. This approach gives us a straightforward way to adopt the notions of probability theory to possibility distributions.

The rest of this paper is organized as follows. In Section 2 we recall the basic notions of possibility correlation, in Section 2 we survey some measures of possibilistic dependence. In Section 4 we discuss the inverse problem, i.e we construct a joint possibility distribution for a given correlation coefficient, in Section 5 we discuss the case when the joint possibility distribution is defined by the weak $t$-norm.

\section{Basic Notions of Possibilistic Correlation}

Definition 2.1. A fuzzy number $A$ is a fuzzy set of $\mathbb{R}$ with a normal, fuzzy convex and continuous membership function of bounded support.

Fuzzy numbers can be viewed as possibility distributions. The concept and some basic properties of joint possibility distribution were introduced in [36].

Definition 2.2. If $A_{1}, \ldots, A_{n}$ are fuzzy numbers, then $C$ is their joint possibility distribution if

$$
A_{i}\left(x_{i}\right)=\max \left\{C\left(x_{1}, \ldots, x_{n}\right) \mid x_{j} \in \mathbb{R}, j \neq i\right\}
$$

holds for all $x_{i} \in \mathbb{R}, i=1, \ldots, n$. Furthermore, $A_{i}$ is called the $i$-th marginal possibility distribution of $C$.

As a special case we define the joint possibility distribution of two fuzzy numbers (see Fig. 1), because we investigate the measures of dependence between pairs of fuzzy numbers.

Definition 2.3. A fuzzy set $C$ in $\mathbb{R}^{2}$ is said to be a joint possibility distribution of fuzzy numbers $A, B$, if it satisfies the relationships

$$
A(x)=\max \{C(x, y) \mid y \in \mathbb{R}\}, \quad \text { and } \quad B(y)=\max \{C(x, y) \mid x \in \mathbb{R}\},
$$

for all $x, y \in \mathbb{R}$. Furthermore, $A$ and $B$ are called the marginal possibility distributions of $C$.

Fuzzy numbers $A_{1}, \ldots, A_{n}$ are said to be non-interactive if their joint possibility distribution $C$ satisfies the relationship

$$
C\left(x_{1}, \ldots, x_{n}\right)=\min \left\{A_{1}\left(x_{1}\right), \ldots, A_{n}\left(x_{n}\right)\right\},
$$

for all $x=\left(x_{1}, \ldots, x_{n}\right) \in \mathbb{R}^{n}$ (see Fig. 2). 
Definition 2.4. A $\gamma$-level set (or $\gamma$-cut) of a possibility distribution $C$ is a non-fuzzy set denoted by $[C]^{\gamma}$ and defined by

$$
[C]^{\gamma}=\left\{\begin{array}{cc}
\left\{(x, y) \in \mathbb{R}^{2} \mid C(x, y) \geq \gamma\right\} & \text { if } \gamma>0 \\
\operatorname{cl}(\operatorname{supp} C) & \text { if } \gamma=0
\end{array}\right.
$$

where $\operatorname{cl}(\operatorname{supp} C)$ denotes the closure of the support of $C$.

\section{Measures of Possibilistic Dependence}

\subsection{Possibilistic Correlation}

Carlsson and Fullér introduced a definition of possibilistic mean and variance [2], and then Fullér and Majlander gave the definition of weighted possibilistic mean and variance [9]. Fullér, Mezei and Várlaki introduced a new definition of possibilistic correlation coefficient [10] between marginal distributions of the joint possibility distribution that improves the earlier definition introduced by Carlsson, Fullér and Majlender [3].

Definition 3.1 (see [10]). Let $f:[0,1] \rightarrow \mathbb{R}$ a non-negative, monotone increasing function with the normalization property $\int_{0}^{1} f(\gamma) \mathrm{d} \gamma=1$. The $f$-weighted possibilistic correlation coefficient of fuzzy numbers $A$ and $B$ (with respect to their joint distribution $C$ ) is defined by

$$
\rho_{f}(A, B)=\int_{0}^{1} \rho\left(X_{\gamma}, Y_{\gamma}\right) f(\gamma) \mathrm{d} \gamma,
$$

where

$$
\rho\left(X_{\gamma}, Y_{\gamma}\right)=\frac{\operatorname{cov}\left(X_{\gamma}, Y_{\gamma}\right)}{\sqrt{\operatorname{var}\left(X_{\gamma}\right)} \sqrt{\operatorname{var}\left(Y_{\gamma}\right)}},
$$

and, where $X_{\gamma}$ and $Y_{\gamma}$ are random variables whose joint distribution is uniform on $[C]^{\gamma}$ for all $\gamma \in[0,1]$, and $\operatorname{cov}\left(X_{\gamma}, Y_{\gamma}\right)$ denotes their probabilistic covariance.

As we can see, the $f$-weighted possibilistic correlation coefficient is the $f$-weighted average of the probabilistic correlation coefficients $\rho\left(X_{\gamma}, Y_{\gamma}\right)$ for all $\gamma \in[0,1]$. Since $f$ is an increasing function, it gives less importance to the lower levels of the possibility distribution. For detailed and illustrated examples see [8][11] and [12].

The range of the $f$-weighted possibilistic correlation coefficient when the marginal possibility distribution have the same membership function was discussed in [19] and [17].

Fuzzy numbers $A$ and $B$ are in perfect correlation [3], if their joint distribution is concentrated along a line (see Fig. 3 and Fig. 4), i.e. if there exist $a, b \in \mathbb{R}, a \neq 0$ such that their joint possibility distribution is

$$
C\left(x_{1}, x_{2}\right)=\left\{\begin{array}{cl}
A\left(x_{1}\right) & \text { if } x_{2}=a x_{1}+b \\
0 & \text { otherwise }
\end{array}\right.
$$




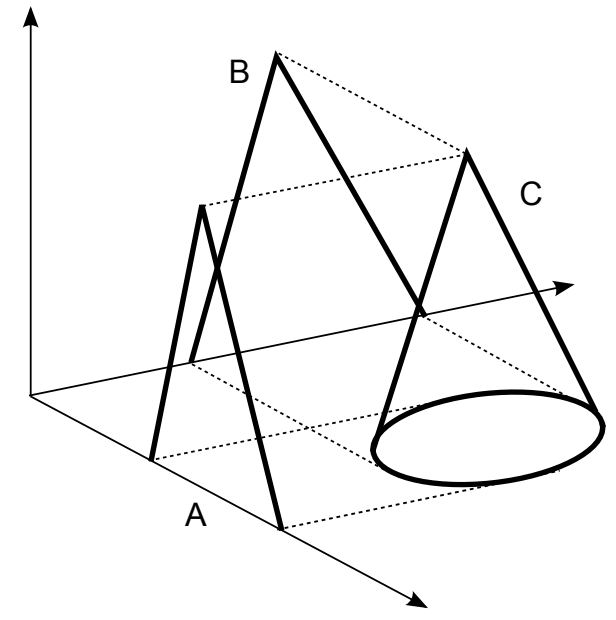

Figure 1: Joint possibility distribution $C$ and its marginal possibility distributions (i.e. projections) fuzzy numbers $A$ and $B$.

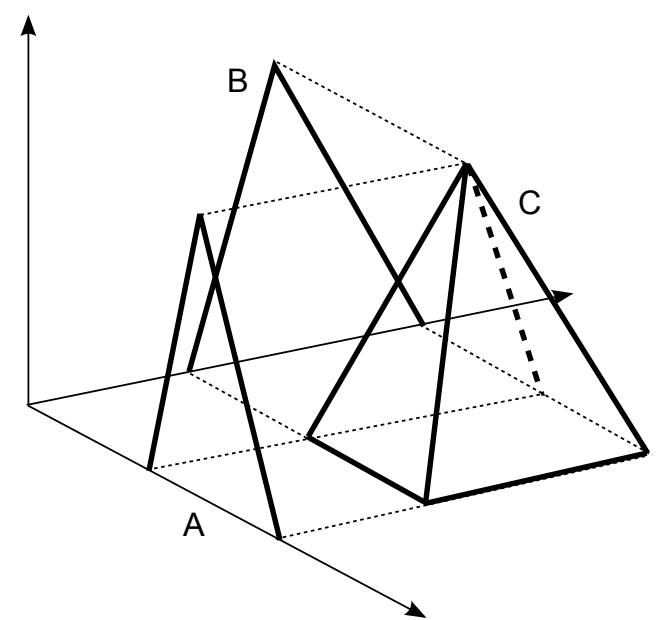

Figure 2: Joint possibility distribution $C$ and its marginal possibility distributions fuzzy numbers $A$ and $B$ when the joint distribution is defined by $\min (A, B)$. In this case $A$ and $B$ are non-interactive which implies $\rho_{f}(A, B)=0$. 


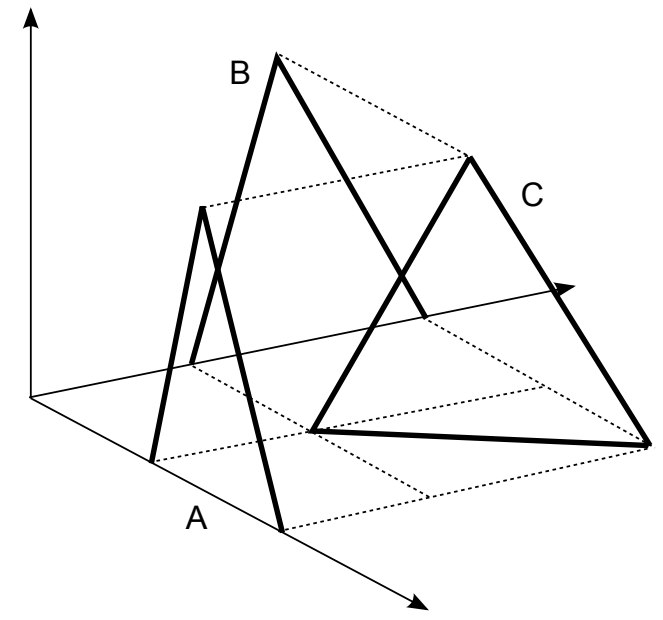

Figure 3: Joint possibility distribution $C$ and its marginal possibility distributions $A$ and $B$ when the joint possibility distribution is defined along a line with positive steepness. This is the case of perfect positive correlation, which implies $\rho_{f}(A, B)=1$.

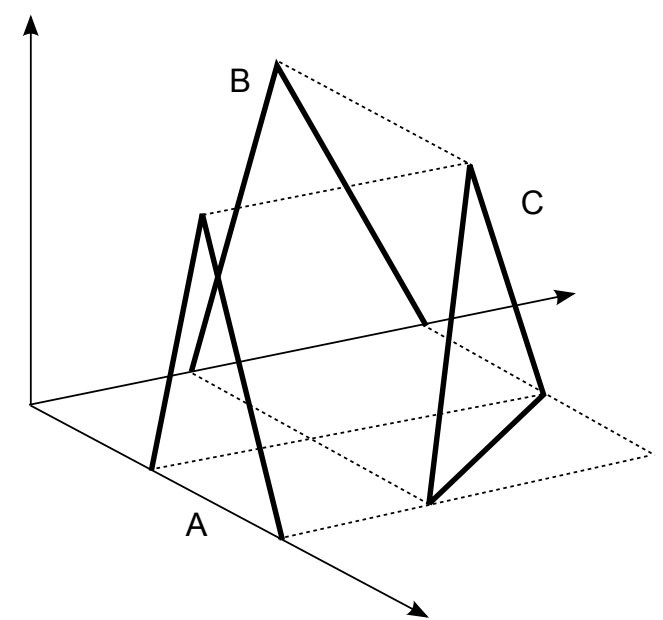

Figure 4: Joint possibility distribution $C$ and its marginal possibility distributions $A$ and $B$ when the joint possibility distribution is defined along a line with negative steepness. This is the case of perfect negative correlation, which implies $\rho_{f}(A, B)=-1$. 
If $A$ and $B$ have a perfect positive (negative) correlation then from $\rho\left(X_{\gamma}, Y_{\gamma}\right)=1$ $\left(\rho\left(X_{\gamma}, Y_{\gamma}\right)=-1\right)$ (see [3] for details), for all $\gamma \in[0,1]$, we get $\rho_{f}(A, B)=1$ $\left(\rho_{f}(A, B)=-1\right)$ for any weighting function $f$.

We should note here that while non-interactivity implies zero correlation, the reverse direction is not necesseraly true, if the value of possibilistic correlation coefficient is zero then this not means automatically non-interactivity. For example, if for every $\gamma,[C]^{\gamma}$ is symmetrical to an axes which parallel with one the coordinate axis then $\operatorname{cov}\left(X_{\gamma}, Y_{\gamma}\right)=0$ and $\rho_{f}(A, B)=0$ for any weighting function $f$ (see [6]).

\subsection{Correlation Ratio Between Fuzzy Numbers}

The correlation ratio $\eta$ was firstly introduced by Karl Pearson [32] as a statistical tool and it was defined to random variables by Kolmogorov [24] as,

$$
\eta^{2}(X \mid Y)=\frac{D^{2}[E(X \mid Y)]}{D^{2}(X)},
$$

where $X$ and $Y$ are random variables. It measures not only a linear, but in general a functional dependence between random variables $X$ and $Y$. If $X$ and $Y$ have a joint probability density function, denoted by $f(x, y)$, then we can compute $\eta^{2}(X \mid Y)$ using the following formulas

$$
E(X \mid Y=y)=\int_{-\infty}^{\infty} x f(x \mid y) \mathrm{d} x
$$

and

$$
D^{2}[E(X \mid Y)]=E(E(X \mid y)-E(X))^{2}
$$

where,

$$
f(x \mid y)=\frac{f(x, y)}{f(y)} .
$$

In 2010 Fullér, Mezei and Várlaki introduced the definition of possibilistic correlation ratio for marginal possibility distributions (see [7]).

Definition 3.2. Let us denote $A$ and $B$ the marginal possibility distributions of a given joint possibility distribution $C$. Then the $f$-weighted possibilistic correlation ratio $\eta_{f}(A \mid B)$ of marginal possibility distribution $A$ with respect to marginal possibility distribution $B$ is defined

$$
\eta_{f}^{2}(A \mid B)=\int_{0}^{1} \eta^{2}\left(X_{\gamma} \mid Y_{\gamma}\right) f(\gamma) \mathrm{d} \gamma
$$

where $X_{\gamma}$ and $Y_{\gamma}$ are random variables whose joint distribution is uniform on $[C]^{\gamma}$ for all $\gamma \in[0,1]$, and $\eta\left(X_{\gamma} \mid Y_{\gamma}\right)$ denotes their probabilistic correlation ratio. 


\subsection{Informational Coefficient of Correlation}

Definition 3.3. For any two continous random variables $X$ and $Y$ (admitting a joint probability density), their mutual information is given by

$$
I(X, Y)=\int_{-\infty}^{\infty} \int_{-\infty}^{\infty} f(x, y) \ln \frac{f(x, y)}{f_{1}(x) \cdot f_{2}(y)} \mathrm{d} x \mathrm{~d} y
$$

where $f(x, y)$ is the joint probability density function of $X$ and $Y$, and $f_{1}(x)$ and $f_{2}(y)$ are the marginal density functions of $X$ and $Y$, respectively.

Definition 3.4. [27] For two random variables $X$ and $Y$, let denote $I(X, Y)$ the mutual information between $X$ and $Y$. Their informational coefficient of correlation is given by

$$
L(X, Y)=\sqrt{1-e^{-2 I(X, Y)}}
$$

Based on the definition above, we can define the following [13][14]:

Definition 3.5. Let us denote $A$ and $B$ the marginal possibility distributions of a given joint possibility distribution $C$. Then the $f$-weighted possibilistic informational coefficient of correlation of marginal possibility distributions $A$ and $B$ is defined by

$$
L(A, B)=\int_{0}^{1} L\left(X_{\gamma}, Y_{\gamma}\right) f(\gamma) \mathrm{d} \gamma
$$

where $X_{\gamma}$ and $Y_{\gamma}$ are random variables whose joint distribution is uniform on $[C]^{\gamma}$ for all $\gamma \in[0,1]$, and $L\left(X_{\gamma}, Y_{\gamma}\right)$ denotes informational coefficient of correlation, and $f$ is a weighting function.

There are several other ways to translate the fundamental notions of probability theory to fuzzy numbers (or possibilistic variables), so there are different interpretations for the mean, variance and covariance of fuzzy numbers. Fuzzy random variables are discussed in [26][34] and [33], the variance of fuzzy random variables in [25][31], variance and covariance studied in [5].

Mean value of fuzzy numbers was defined in [4] and [20], the notion of independence is studied in [1], [21] and [35], and with applications in [29], [30].

Liu and Kao [28] used fuzzy measures to define a fuzzy correlation coefficient of fuzzy numbers and they formulated a pair of nonlinear programs to find the $\alpha$-cut of this fuzzy correlation coefficient, then, in a special case, Hong [22] showed an exact calculation formula for this fuzzy correlation coefficient.

In [15] Fullér et al. introduced a method as a generalization of the concept described in [10]. Here the $\gamma$-level sets are equipped with non-uniform probability distribution, whose density function is derived from the joint possibility distribution. 


\section{Joint Possibility Distribution for Given Correlation}

In this section we show a simple way to construct a joint possibility distribution (and in this way marginal possibility distributions) for a given value of the possibilistic correlation coefficient. We recall the fact in probability theory that for any value between -1 and 1 there exists a 2-dimensional Gaussian distribution whose marginal distributions has this value as correlation coefficient between them (for other types of distributions it is not necesseraly true).

Let the required value of the possibilistic correlation coefficient be $\rho$. Define the joint possibilistic distribution as follows:

$$
C(x, y)=\exp \left(\frac{-1}{2\left(1-\rho^{2}\right)} \cdot\left(x^{2}-2 \rho x y+y^{2}\right)\right)
$$

The $\gamma$-level set (remember that $0<\gamma \leq 1$, so $\ln \gamma \leq 0$ ):

$$
[C]^{\gamma}=\left\{(x, y) \in \mathbb{R}^{2} \mid x^{2}-2 \rho x y+y^{2} \leq-2\left(1-\rho^{2}\right) \cdot \ln \gamma\right\}
$$

The $\gamma$-level set is a (maybe skew) ellipse, whose upper and lower curves are

$$
\begin{aligned}
& y_{1}=\rho x+\sqrt{1-\rho^{2}} \cdot \sqrt{-2 \ln \gamma-x^{2}} \\
& y_{2}=\rho x-\sqrt{1-\rho^{2}} \cdot \sqrt{-2 \ln \gamma-x^{2}}
\end{aligned}
$$

The area of the $\gamma$-levels set is $T_{\gamma}=-2 \pi \sqrt{1-\rho^{2}} \cdot \ln \gamma$. According to the definition of possibilistic correlation coefficient, we define a two dimensional uniform distribution on the $\gamma$-level set, so its density function is

$$
f(x, y)=\left\{\begin{array}{cl}
\frac{1}{T_{\gamma}} & \text { if }(x, y) \in[C]^{\gamma} \\
0 & \text { otherwise }
\end{array}\right.
$$

$X_{\gamma}$ and $Y_{\gamma}$ are its marginal random variables. The marginal density function of $X_{\gamma}$ ( $Y_{\gamma}$ has the same one):

$$
f_{1}(x)=\left\{\begin{array}{cl}
\frac{-\sqrt{-2 \ln \gamma-x^{2}}}{\pi \cdot \ln \gamma} & \text { if }-\sqrt{-2 \ln \gamma}<x<\sqrt{-2 \ln \gamma} \\
0 & \text { otherwise }
\end{array}\right.
$$

The expected values are

$$
\begin{aligned}
M\left(X_{\gamma}\right) & =M\left(Y_{\gamma}\right)=0 \\
M\left(X_{\gamma}^{2}\right) & =M\left(Y_{\gamma}^{2}\right)=\frac{-\ln \gamma}{2} \\
M\left(X_{\gamma} \cdot Y_{\gamma}\right) & =\frac{-\rho \cdot \ln \gamma}{2}
\end{aligned}
$$


So the correlation coefficient at level $\gamma$ :

$$
\rho\left(X_{\gamma}, Y_{\gamma}\right)=\frac{\operatorname{cov}\left(X_{\gamma}, Y_{\gamma}\right)}{\sqrt{\operatorname{var}\left(X_{\gamma}\right)} \sqrt{\operatorname{var}\left(Y_{\gamma}\right)}}=\frac{-\rho \cdot \ln \gamma / 2}{-\ln \gamma / 2}=\rho
$$

Since the value of $\rho$ not depends on $\gamma$, the value of possibilistic correlation equals this value:

$$
\rho_{f}(A, B)=\int_{0}^{1} \rho\left(X_{\gamma}, Y_{\gamma}\right) f(\gamma) \mathrm{d} \gamma=\rho \int_{0}^{1} f(\gamma) \mathrm{d} \gamma=\rho
$$

Note 4.1. In fact the starting point was a two dimensional Gaussian probability density function:

$$
f(x, y)=\frac{1}{2 \pi \sqrt{1-\rho^{2}}} \cdot \exp \left(\frac{-1}{2\left(1-\rho^{2}\right)} \cdot\left(x^{2}-2 \rho x y+y^{2}\right)\right)
$$

, where $\rho$ is the correlation coefficient between the marginal random variables. So the result we get tells us that the possibilistic and probabilistic correlation coefficient could be the same for certain cases.

\section{Correlation Coefficient for $t$-norm Defined Joint Distributions}

An interestinq question is the range or behaviour of the possibilistic correlation coefficient when the joint possibility distribution has a special structure, i.e. it is defined by a $t$-norm. According to our best knowledge there are no simple general results to this problem. For the most widely used $t$-norm, the minimum $t$-norm the answer is straightforward, since this is the case when the marginal distributions (fuzzy numbers) are in non-interactive relation and this fact ensures zero correlation coefficient.

The case when the joint possibility distribution is defined by the product $t$-norm was discussed in [12], where the authors pointed out that the value of the possibilistic correlation falls between $-1 / 2$ and $1 / 2$, including the limits.

Well-known that the following inequality holds for any $t$-norm:

$$
T_{w}(a, b) \leq t(a, b) \leq \min (a, b)
$$

where $T_{w}$ denotes the weak (or drastic) $t$-norm:

$$
T_{w}(a, b)=\left\{\begin{array}{cl}
\min (a, b) & \text { if } \max (a, b)=1, \\
0 & \text { otherwise. }
\end{array}\right.
$$

In the following we give strict bounds for the possibilistic correlation coefficients when the joint distribution $C(x, y)=T_{w}(A(x), B(y))$, where $A$ and $B$ are the marginal distributions. 

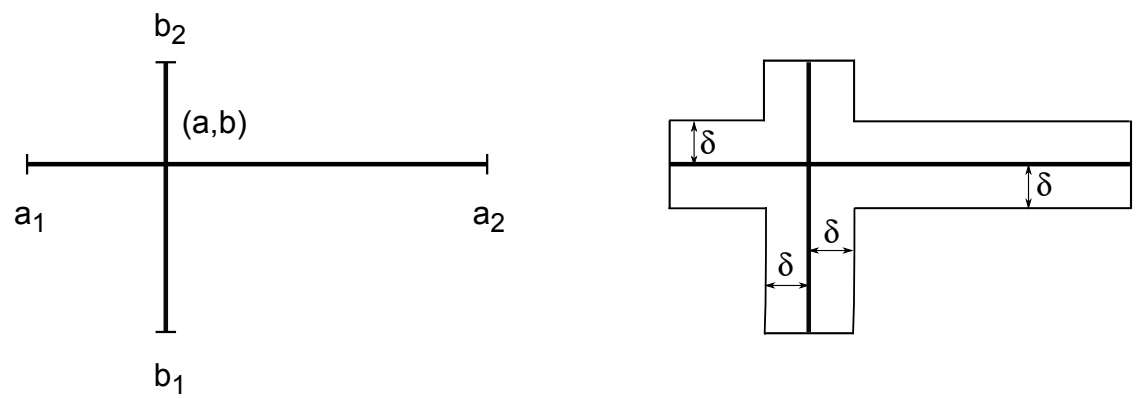

Figure 5: The $\gamma$ level set of the joint distribution, when $C(x, y)=T_{w}(A(x), B(y))$ (left), and its $\delta$ neighborhood (right).

Let us denote the core of fuzzy number $A$ by $a$, the core of $B$ by $b$, the $\gamma$ level sets by $\left[a_{1}(\gamma), a_{2}(\gamma)\right]$ and $\left[b_{1}(\gamma), b_{2}(\gamma)\right]$, respectively. For simplicity we use the notations: $a_{1}=a_{1}(\gamma), a_{2}=a_{2}(\gamma), b_{1}=b_{1}(\gamma)$ and $b_{2}=b_{2}(\gamma)$. The $\gamma$-level sets $\left([C]^{\gamma} \mathbf{s}\right)$ of the joint distribution are (not necessarily symmetric) cross-shaped domains (see Fig. 5). The correlation coefficient for this domain is determined as the limit of the correlation coefficient computed for the $\delta$ neighborhood $\left([C]_{\delta}^{\gamma}\right.$ ) (see Fig. 5). Since the correlation coefficient is invariant under shifting and scaling (multiplying by a positive constant) of the marginal distributions, without loss of generality we can assume that $a=b=0$, and $-1 \leq a_{1}, b_{1} \leq 0,0 \leq a_{2}, b_{2} \leq 1$, such that at least one of the following conditions hold: $a_{1}=-1, b_{1}=-1, a_{1}=-1, b_{2}=1, a_{2}=1, b_{2}=1$ or $a_{2}=1, b_{1}=-1$. These conditions are always feasible: we shift the cores to the origin, then rescale $A$ by $\max \left\{\left|a_{1}\right|, a_{2}\right\}$ and $B$ by $\max \left\{\left|b_{1}\right|, b_{2}\right\}$.

$X_{\gamma}$ and $Y_{\gamma}$ are random variables, whose joint distribution is uniform on $[C]_{\delta}^{\gamma}$, the corresponding random variables for $[C]^{\gamma}$ are $X_{\gamma}^{\prime}$ and $Y_{\gamma}^{\prime}$.

The probability density function of $X_{\gamma}\left(Y_{\gamma}\right.$ has the same with appropriate modification of the parameters):

$$
f_{1}(x)=\left\{\begin{array}{cl}
\frac{1}{T} \cdot 2 \delta & , \text { if } a_{1}<x<-\delta \\
\frac{1}{T} \cdot\left(b_{2}-b_{1}\right) & , \text { if }-\delta<x<\delta \\
\frac{1}{T} \cdot 2 \delta & , \text { if } \delta<x<a_{2} .
\end{array}\right.
$$

where $T=\left(a_{2}-a_{1}\right) \cdot 2 \delta+\left(b_{2}-b_{1}\right) \cdot 2 \delta-4 \delta^{2}$ denotes the area of $[C]_{\delta}^{\gamma}$. 
Computing the expected values and after some simplifications we get:

$$
\begin{aligned}
& M\left(X_{\gamma}\right)=\frac{a_{2}^{2}-a_{1}^{2}}{2\left(a_{2}-a_{1}+b_{2}-b_{1}\right)-4 \delta} \\
& M\left(X_{\gamma}^{\prime}\right)=\lim _{\delta \rightarrow 0} M\left(X_{\gamma}\right)=\frac{a_{2}^{2}-a_{1}^{2}}{2\left(a_{2}-a_{1}+b_{2}-b_{1}\right)} \\
& M\left(X_{\gamma}^{2}\right)=\frac{2}{3} \cdot \frac{a_{2}^{3}-a_{1}^{3}+\left(b_{2}-b_{1}\right) \delta^{2}-2 \delta^{3}}{2\left(a_{2}-a_{1}+b_{2}-b_{1}\right)-4 \delta} \\
& M\left(X_{\gamma}^{\prime 2}\right)=\lim _{\delta \rightarrow 0} M\left(X_{\gamma}^{2}\right)=\frac{2}{3} \cdot \frac{a_{2}^{3}-a_{1}^{3}}{2\left(a_{2}-a_{1}+b_{2}-b_{1}\right)}
\end{aligned}
$$

Similar expressions hold for $Y_{\gamma}$ with appropriate modification of the parameters, of course. The expected value of the product:

$$
M\left(X_{\gamma} \cdot Y_{\gamma}\right)=0 \quad \Rightarrow \quad M\left(X_{\gamma}^{\prime} \cdot Y_{\gamma}^{\prime}\right)=0
$$

The correlation coefficient between $X_{\gamma}^{\prime}$ and $Y_{\gamma}^{\prime}$ :

$$
\rho\left(X_{\gamma}^{\prime}, Y_{\gamma}^{\prime}\right)=\frac{\operatorname{cov}\left(X_{\gamma}^{\prime}, Y_{\gamma}^{\prime}\right)}{\sqrt{\operatorname{var}\left(X_{\gamma}^{\prime}\right)} \cdot \sqrt{\operatorname{var}\left(Y_{\gamma}^{\prime}\right)}}
$$

where

$$
\begin{aligned}
\operatorname{cov}\left(X_{\gamma}^{\prime}, Y_{\gamma}^{\prime}\right) & =M\left(X_{\gamma}^{\prime} \cdot Y_{\gamma}^{\prime}\right)-M\left(X_{\gamma}^{\prime}\right) \cdot M\left(Y_{\gamma}^{\prime}\right)=\frac{-\left(a_{2}^{2}-a_{1}^{2}\right)\left(b_{2}^{2}-b_{1}^{2}\right)}{4\left(a_{2}-a_{1}+b_{2}-b_{1}\right)^{2}} \\
\operatorname{var}\left(X_{\gamma}^{\prime}\right) & =\frac{\frac{4}{3} \cdot\left(a_{2}^{3}-a_{1}^{3}\right) \cdot\left(a_{2}-a_{1}+b_{2}-b_{1}\right)-\left(a_{2}^{2}-a_{1}^{2}\right)^{2}}{4\left(a_{2}-a_{1}+b_{2}-b_{1}\right)^{2}} \\
\operatorname{var}\left(Y_{\gamma}^{\prime}\right) & =\frac{\frac{4}{3} \cdot\left(b_{2}^{3}-b_{1}^{3}\right) \cdot\left(a_{2}-a_{1}+b_{2}-b_{1}\right)-\left(b_{2}^{2}-b_{1}^{2}\right)^{2}}{4\left(a_{2}-a_{1}+b_{2}-b_{1}\right)^{2}}
\end{aligned}
$$

We prove that the value of the above correlation coefficient always falls between $-3 / 5$ and $3 / 5$. Let's consider the case when $a_{2}=1$ and $b_{2}=1$ (the estimation works quite similarly for the other three cases). We give a lower estimation for the variances using the fact that $-1 \leq a_{1} \leq 0$ and $-1 \leq b_{1} \leq 0$, so we get an upper estimation for the 
correlation coefficient. The numerator of $\operatorname{var}\left(X_{\gamma}^{\prime}\right)$ :

$$
\begin{aligned}
& \frac{4}{3} \cdot\left(1-a_{1}^{3}\right) \cdot\left(2-a_{1}-b_{1}\right)-\left(1-a_{1}^{2}\right)^{2} \\
& \geq \frac{4}{3} \cdot\left(1-a_{1}^{2}\right)^{2} \cdot\left(2-a_{1}-b_{1}\right)-\left(1-a_{1}^{2}\right)^{2} \\
& =\left(1-a_{1}^{2}\right)^{2} \cdot\left[\frac{4}{3} \cdot\left(2-a_{1}-b_{1}\right)-1\right] \\
& \geq\left(1-a_{1}^{2}\right)^{2} \cdot\left[\frac{4}{3} \cdot 2-1\right]=\left(1-a_{1}^{2}\right)^{2} \cdot \frac{5}{3}
\end{aligned}
$$

Applying this result we get that

$$
\operatorname{var}\left(X_{\gamma}^{\prime}\right) \geq \frac{\left(1-a_{1}^{2}\right)^{2} \cdot \frac{5}{3}}{4\left(a_{2}-a_{1}+b_{2}-b_{1}\right)^{2}}
$$

So we get the following bounds for the square of the correlation coefficient:

$$
\rho^{2} \leq \frac{\left(1-a_{1}^{2}\right)^{2}\left(1-b_{1}^{2}\right)^{2}}{\left(1-a_{1}^{2}\right)^{2} \cdot \frac{5}{3} \cdot\left(1-b_{1}^{2}\right)^{2} \cdot \frac{5}{3}}=\frac{9}{25}
$$

which yields:

$$
-3 / 5 \leq \rho \leq 3 / 5
$$

These bounds are strict, since

- if $a_{2}=b_{2}=0$ and $a_{1}=b_{1}=-1$, then $\rho=-3 / 5$;

- if $a_{2}=b_{1}=0$ and $a_{1}=-1, b_{2}=1$, then $\rho=3 / 5$.

Remember that the possibilistic correlation coefficient was defined as the weighted average of probabilistic correlation coefficients over the $\gamma$ levels. We proved that for every $\gamma$ level set $-3 / 5 \leq \rho\left(X_{\gamma}, Y_{\gamma}\right) \leq 3 / 5$, so these inequality holds for the possibilistic correlation coefficient for any weighting function $f$ :

$$
-3 / 5 \leq \rho_{f}(A, B) \leq 3 / 5
$$

Our numerical and theoretical investigations done so far led us to a conjecture that the weak $t$-norm has a kind of boundary role here, which is still an open problem:

Question 5.1. Is it true that for any joint possibility distribution defined by a t-norm, the possibilistic correlation coefficient falls between $-3 / 5$ and $3 / 5$ ? 


\section{Conclusions}

We briefly surveyed the developments of probability related $\gamma$-level based possibilistic measures of dependence. This level-based approach gives a useful tool to directly generalize the notions of probability theory to possibilistic variables and it may make a bridge between possibilistic and probabilistic ways of thinking. Although this connection gaves us a chance to adopt the results of probability theory, there are still many open questions.

We gave a short general solution to the inverse problem, namely we showed a family of joint possibility distributions to any given value of correlation. We determined the range of possibilistic correlation coefficient when the joint distribution is defined by the weak $t$-norm. Finally, we stated an open problem for the family of $t$-norm defined joint possibility distribution.

\section{Acknowledgement}

This work was supported by FIEK program (Center for Cooperation between Higher Education and the Industries at the Széchenyi István University, GINOP-2.3.4-152016-00003).

\section{References}

[1] L. M. de Campos, J. F. Huete, Independence concepts in possibility theory: Part I, Fuzzy Sets and Systems 103 (1999), pp. 127-152.

Part II. Fuzzy Sets and Systems 103 (1999), pp. 487-505.

[2] C. Carlsson, R. Fullér, On possibilistic mean and variance of fuzzy numbers, Fuzzy Sets and Systems 122 (2001), pp. 315-326.

[3] C. Carlsson, R. Fullér and P. Majlender, On possibilistic correlation, Fuzzy Sets and Systems, 155(2005) 425-445. doi: 10.1016/j.fss.2005.04.014

[4] D. Dubois and H. Prade, The mean value of a fuzzy number, Fuzzy Sets and Systems, 24(1987), pp. 279-300. doi: 10.1016/0165-0114(87)90028-5

[5] Y. Feng, L. Hu, H. Shu, The variance and covariance of fuzzy random variables and their applications, Fuzzy Sets and Systems 120 (2001), pp. 487-497.

[6] R. Fullér and P. Majlender, On interactive possibility distributions, in: V.A. Niskanen and J. Kortelainen eds., On the Edge of Fuzziness, Studies in Honor of Jorma K. Mattila on His Sixtieth Birthday, Acta universitas Lappeenrantaensis, No. 179, 2004 61-69.

[7] R. Fullér, J. Mezei and P. Várlaki, A Correlation Ratio for Possibility Distributions, in: E. Hüllermeier, R. Kruse, and F. Hoffmann (Eds.): Computational 
Intelligence for Knowledge-Based Systems Design, Proceedings of the International Conference on Information Processing and Management of Uncertainty in Knowledge-Based Systems (IPMU 2010), June 28 - July 2, 2010, Dortmund, Germany, Lecture Notes in Artificial Intelligence, vol. 6178(2010), SpringerVerlag, Berlin Heidelberg, pp. 178-187. doi: 10.1007/978-3-642-14049-5_19

[8] R. Fullér, J. Mezei and P. Várlaki, Some Examples of Computing the Possibilistic Correlation Coefficient from Joint Possibility Distributions, in: Imre J. Rudas, János Fodor, Janusz Kacprzyk eds., Computational Intelligence in Engineering, Studies in Computational Intelligence Series, vol. 313/2010, Springer Verlag, [ISBN 978-3-642-15219-1], pp. 153-169. doi: 10.1007/978-3-642-15220-7_13

[9] R. Fullér, P. Majlender, On weighted possibilistic mean and variance of fuzzy numbers, Fuzzy Sets and Systems 136 (2003), pp. 363-374.

[10] R. Fullér, J. Mezei, P. Várlaki, An improved index of interactivity for fuzzy numbers, Fuzzy Sets and Systems 165 (2011), pp. 50-60.

[11] R. Fullér, I. Á. Harmati, J.Mezei, P. Várlaki, On Possibilistic Correlation Coefficient and Ratio for Fuzzy Numbers, in: Recent Researches in Artificial Intelligence, Knowledge Engineering \& Data Bases, 10th WSEAS International Conference on Artificial Intelligence, Knowledge Engineering and Data Bases, February 20-22, 2011, Cambridge, UK, WSEAS Press, [ISBN 978-960-474237-8], pp. 263-268.

[12] R. Fullér, I. Á. Harmati, P. Várlaki, On Possibilistic Correlation Coefficient and Ratio for Triangular Fuzzy Numbers with Multiplicative Joint Distribution, in: Proceedings of the Eleventh IEEE International Symposium on Computational Intelligence and Informatics (CINTI 2010), November 1820, 2010, Budapest, Hungary, [ISBN 978-1-4244-9278-7], pp. 103-108. DOI 10.1109/CINTI.2010.5672266

[13] R. Fullér, I. Á. Harmati, P. Várlaki, I. Rudas, On Informational Coefficient of Correlation for Possibility Distributions, Recent Researches in Artificial Intelligence and Database Management, Proceedings of the 11th WSEAS International Conference on Artificial Intelligence, Knowledge Engineering and Data Bases (AIKED '12), February 22-24, 2012, Cambridge, UK, [ISBN 978-1-61804-0688], pp. 15-20.

[14] R. Fullér, I. Á. Harmati, P. Várlaki, I. Rudas, On Weighted Possibilistic Informational Coefficient of Correlation, International Journal of Mathematical Models and Methods in Applied Sciences, 6(2012), issue 4, pp. 592-599.

[15] R. Fullér, I. Á. Harmati, P. Várlaki, Probabilistic Correlation Coefficients for Possibility Distributions, Fifteenth IEEE International Conference on Intelligent Engineering Systems 2011 (INES 2011), June 23-25, 2011, Poprad, Slovakia, [ISBN 978-1-4244-8954-1], pp. 153-158. DOI 10.1109/INES.2011.5954737 
[16] R. Fullér, I. Á. Harmati, P. Várlaki, On Probabilistic Correlation Coefficients for Fuzzy Numbers, in: Aspects of Computational Intelligence: Theory and Applications: Revised and Selected Papers of the 15th IEEE International Conference on Intelligent Engineering Systems 2011, INES 2011, Topics in Intelligent Engineering and Informatics series, vol. 2/2013, Springer Verlag, [ISBN:978-3-64230667-9], 2013. pp. 249-263. DOI 10.1007/978-3-642-30668-6_17

[17] R. Fullér, I. Á. Harmati, On the lower limit of possibilistic correlation coefficient for identical marginal distributions, 8th European Symposium on Computational Intelligence and Mathematics (ESCIM 2016), 2016, Cádiz, pp. 37-42.

[18] H. Gebelein, Das satistische Problem der Korrelation als Variations- und Eigenwertproblem und sein Zusammenhang mit der Ausgleichungsrechnung, Zeitschrift fr angew. Math. und Mech., 21 (1941), pp. 364-379.

[19] I. Á. Harmati, A note on f-weighted possibilistic correlation for identical marginal possibility distributions, Fuzzy Sets and Systems, 165(2011), pp. 106110. doi: 10.1016/j.fss.2010.11.005

[20] S. Heilpern, The expected value of a fuzzy number, Fuzzy Sets and Systems 47 (1992), pp. 81-86.

[21] E. Hisdal, Conditional possibilities independence and noninteraction, Fuzzy Sets and Systems 1 (1978), pp. 283-297.

[22] D.H. Hong, Fuzzy measures for a correlation coefficient of fuzzy numbers under $T_{W}$ (the weakest t-norm)-based fuzzy arithmetic operations, Information Sciences, 176(2006), pp. 150-160.

[23] E.T. Jaynes, Probability Theory : The Logic of Science, Cambridge University Press, 2003.

[24] A.N. Kolmogorov, Grundbegriffe der Wahrscheinlichkeitsrechnung, Julius Springer, Berlin, 1933, 62 pp.

[25] R. K. Körner, On the variance of fuzzy random variables, Fuzzy Sets and Systems 92 (1997), pp. 8393.

[26] H. Kwakernaak, Fuzzy random variables-I. Definitions and theorems, Information Sciences 15 (1978), pp. 1-29.

[27] E. H. Linfoot, An informational measure of correlation, Information and Control, Vol.1, No. 1 (1957), pp. 85-89.

[28] S.T. Liu, C. Kao, Fuzzy measures for correlation coefficient of fuzzy numbers, Fuzzy Sets and Systems, 128(2002), pp. 267-275.

[29] X. Li, B. Liu, The independence of fuzzy variables with applications, International Journal of Natural Sciences \& Technology 1 (2006), pp. 95-100. 
Fullér et al. On Measures of Dependence Between Possibility Distributions

[30] Y. K. Liu, J. Gao, The independence of fuzzy variables with applications to fuzzy random optimization, International Journal of Uncertainty, Fuzziness \& Knowledge-Based Systems 15 (2007), pp. 1-19.

[31] W. Näther, A. Wünsche, On the Conditional Variance of Fuzzy Random Variables, Metrika 65 (2007), pp. 109-122.

[32] K. Pearson, On a New Method of Determining Correlation, when One Variable is Given by Alternative and the Other by Multiple Categories, Biometrika, Vol. 7, No. 3 (Apr., 1910), pp. 248-257.

[33] M. L. Puri, D. A. Ralescu, Fuzzy random variables, Journal of Mathematical Analysis and Applications 114 (1986), pp. 409-422.

[34] A. F. Shapiro, Fuzzy random variables, Insurance: Mathematics and Economics 44 (2009), pp. 307314.

[35] S. Wang, J. Watada, Some properties of $T$-independent fuzzy variables, Mathematical and Computer Modelling 53 (2011), pp. 970-984.

[36] L. A. Zadeh, Concept of a linguistic variable and its application to approximate reasoning I, II, III Information Sciences 8 (1975), pp. 199-249, pp. 301-357;

L. A. Zadeh, Concept of a linguistic variable and its application to approximate reasoning I, II, III Information Sciences 9 (1975), pp. 43-80. 